\title{
Numerical Simulations and Control of Offshore Energy Harvesting Using Piezoelectric Materials in a Portal Frame Structure
}

\author{
Wagner B. Lenz $\mathbb{D}$, Mauricio A. Ribeiro $\mathbb{D}$, Rodrigo T. Rocha $\mathbb{D}$, \\ Jose M. Balthazar $i D$, and Angelo M. Tusset \\ Department of Electrical Engineering, Federal University of Technology-Parana (UTFPR), Ponta Grossa, PR, Brazil \\ Correspondence should be addressed to Angelo M. Tusset; tusset@utfpr.edu.br
}

Received 21 December 2020; Revised 20 April 2021; Accepted 21 May 2021; Published 3 June 2021

Academic Editor: Mi-An Xue

Copyright ( $\odot 2021$ Wagner B. Lenz et al. This is an open access article distributed under the Creative Commons Attribution License, which permits unrestricted use, distribution, and reproduction in any medium, provided the original work is properly cited.

\begin{abstract}
Buoy systems are an alternative for micropowering small devices in remote locations. Portal frames are very useful to harvest the energy of the waves into usable energy. Thus, using the current models for a portal frame in the literature and the spectrum of available energy in sea waves, a nonlinear mathematical model accounting for the coupling of a nonlinear piezoelectric material is considered. The neighbour of selected variables is analyzed and then optimized by a process utilizing the particle swarm optimization (PSO) algorithm. Furthermore, an optimal control using the linear-quadratic regulator (LQR) controller is applied to control the load resistance of the piezoelectric circuit. The optimization process and the LQR show to be effective. The results show a general gain due to optimization and a relatively small gain using the controller.
\end{abstract}

\section{Introduction}

Due to the large coverage area of the ocean on the Earth's surface, offshore energy sources have been very attractive, bringing the attention of scientists and engineers of the energy field. This enormous interest stands out for the power that can be used as a source for consumption in different ways [1-3]. This energy is determined by the mechanical waves formed by the ocean's water motion. According to $[1,2]$, it is estimated that approximately 850 TWh of electricity is generated with the conversion of this movement. In this way, several devices work with the amplitude of the tides, such as those that lift the floats that work with the sea waves generated by the wind, devices that work with the torsion system, and the ones that work by creating air pressure and thus generating electricity [4].

The authors in $[5,6]$ utilized an air turbine actuated by the displacement through the oscillating of the ocean waves for energy generation, where such energy is stored in the form of compressed air. The authors in [7] converted the ocean wave energy through oscillating bodies coupled to a linear electrical generator. On the same hand, the authors in [8] considered the overtopping floating structures as the Wave Dragon energy converter.
Therefore, with the high demand for energy consumption nowadays, the need for new power sources has been growing in the last decades, especially in remote locations that make the energy conversion on site a more attractive source, and for lowpower devices, such as underwater sensors.

The use of solar panels is an alternative power source that has been used for enhancing offshore energy harvesting [9]. However, it has a discontinuous load capacity, and the panels are not recyclable and required frequent cleaning.

In an attempt to improve the offshore energy conversion, the authors in [10] presented an ocean wave energy harvesting system accounting for a direct current (DC) power generator attached at the middle top of a floating platform along with a pendulum connected to the generator's shaft. It was considered that the ocean wave motion swings the platform in the vertical direction, inducing the pendulum rotation. Consequently, electrical energy is provided due to the DC power generator. For optimizing the harvested power, the pendulum parameters were analyzed by using the PSO algorithm taking into account the mass and length variation of the pendulum. In addition, the ocean wave amplitude and frequency are also considered. The numerical results showed an efficient energy conversion with the use of the DC power supply. 
Piezoelectric materials are also an alternative for enhancing offshore energy harvesting. A floating platform is generally induced to vibrate due to the ocean waves, which makes the piezoelectric material an intelligent strategy.

These materials have been studied and used as shown in [11]. These authors have explored piezoelectric materials for energy harvesting due to the wide range of frequency utility (including high frequency); they are of low-power consumption, ease of application, and very low cost.

In [12], a piezoelectric energy harvester based on a polygon-shaped cantilever array is considered. The authors employed a multifrequency operating principle for eight cantilevers with the irregular design of the cross-sectional area. A novel V-shaped vibration energy harvester based on the conventional piezoelectric bimorph cantilevered structure is explored in [13]. Experimental results showed that the $\mathrm{V}$-shaped energy harvester improved the frequency response characteristic and the output performance of the electrical energy. In [14], three different kinds of energy harvesting generators are designed: one considering the monostable piezoelectric cantilever beam structure and the other two considering bistable piezoelectric cantilever beam structures. Numerical and experimental analyses were considered for power generation and dynamic behaviors of the different structures. In $[15,16]$, the introduction of a nonlinear piezoelectric coupling in a portal frame accounting for the lateral motion of the structure is considered. Numerical results showed that energy harvesting can be influenced by nonlinear parameters of the piezoelectric model.

Furthermore, the installation of the piezoelectric sensor to the seaward position in an existing coastal structure for generating energy from sea waves is carried out in [17]. The authors used a wave breaker along with the structure. Experimental results showed correlations of generation volume, and wave conditions have been found.

Another form of application is utilizing the piezoelectric material as an actuator in flexible elements to activate or prevent deflections on it. In this case, the reverse effect is a desired effect and could be used with the genetic algorithm (GA), further improving the location and resizing the piezoelectric [18]. With that, the State-Dependent Riccati Equation (SDRE) controller is used for driving the piezoelectric material to a more efficient behavior for energy harvesting [19], which may pump vibration energy into the system to control its movement.

In [9], a combination of a fuzzy-PID controller and an LQR controller was used for determining the movement of a plate. The authors concluded that both strategies are valid and robust to control the experiment and better evaluate the performance of the structure. In addition, details about the difficulty and complexity of using piezoelectric actuators in structures were discussed.

In the offshore field, as the buoy is in constant motion, it is a logical solution to harvest this vibration energy. Due to the power output, the combination of various piezoelectric materials further increases reliability. Piezoelectric material is reliable and is not only used for collecting energy. This material is present in humidifiers, printers, and high-performance diesel engines, proving its applicability in several fields and a consolidating technology.
Therefore, this work proposes the analysis of the energy harvesting of an offshore system containing a portal frame structure along with a piezoelectric material for energy conversion. The mathematical modelling of the system is carried out. Particle swarm optimization (PSO) is considered to optimize the energy production of the system. In addition, the LQR control technique is used for maintaining the energy conversion at its peak production. The parametrical analysis of the system is carried out throughout numerical simulations accounting for the Lyapunov exponent study, which is used for tracking the dynamic behavior of the structure to maximize the energy conversion.

The rest of the work is organized in the following way. Section 2 describes the mathematical model and the structure in question. Section 3 describes the PSO algorithm. Section 4 presents the LQR optimal control. Section 5 shows the numerical simulations of the optimization of the parameters using the PSO, the project of the control, and dynamical analysis. Finally, Section 6 presents the conclusions of this study.

\section{The Buoy System}

Buoy systems are floating devices attached to the seabed that, by themselves, can be used for generating electrical energy. Most of the buoy systems are used for large macroenergy generation. However, in this case, we are interested in micropower harvesting.

In Figures 1(a)-1(c), a simple bounce system, a portal frame structure with a tuned-mass-damper (TMD) system on top of it, and the lumped parameter scheme of the system are shown, respectively $[20,21]$. Note that the portal frame system (Figures 1 (b) and 1(c)) is located on $A_{1}$.

2.1. Mathematical Model. The mathematical model of the portal frame represented in Figure 1(c) is a complex iteration between the masses $m_{1}$ and $m_{2}$. The mode is represented as a nonlinear dynamic model modelled through Newton's second law, along with the differential equation that describes the voltage of the piezoelectric material depending on the deflection of the structure. The equations of motion of the system are given by

$$
\begin{aligned}
\ddot{X}_{1}= & \frac{1}{m_{1}}\left(-\frac{\sigma}{C} q-k_{l 1} \Delta_{1}-k_{n l 1} \Delta_{1}^{3}-b_{1} \dot{\Delta}_{1}\right. \\
& \left.+k_{2} \Delta_{2}+b_{2} \dot{\Delta}_{2}\right) \\
\ddot{X}_{2}= & \frac{1}{m_{2}}\left(-k_{2} \Delta_{2}-b_{2} \dot{\Delta}_{2}\right) \\
R \dot{q}-\left(\frac{\sigma}{C} X_{1}+\frac{q}{C}\right)= & 0
\end{aligned}
$$

where $\quad \Delta_{1}=X_{1}-X_{w}, \Delta_{2}=X_{2}-X_{1}, \dot{\Delta}_{1}=\dot{X}_{1}-\dot{X}_{w}, \dot{\Delta}_{2}=$ $\dot{X}_{2}-\dot{X}_{1}$.

The nonlinear piezoelectric coupling is defined as follows:

$$
\sigma=\sigma_{1}\left(1+\sigma_{2}\left|X_{1}\right|\right)
$$




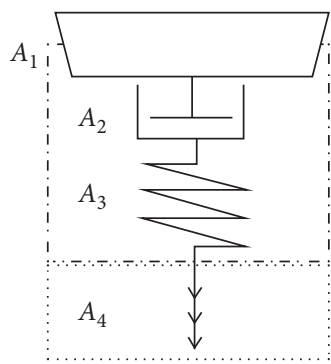

(a)

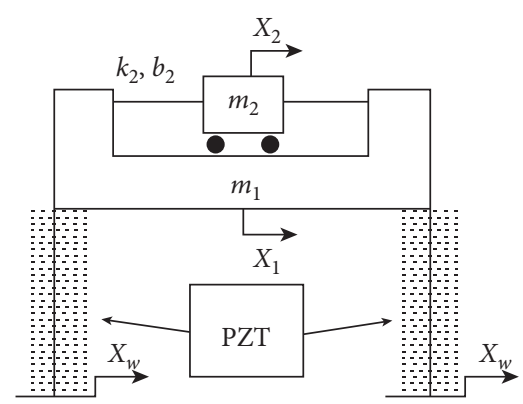

(b)

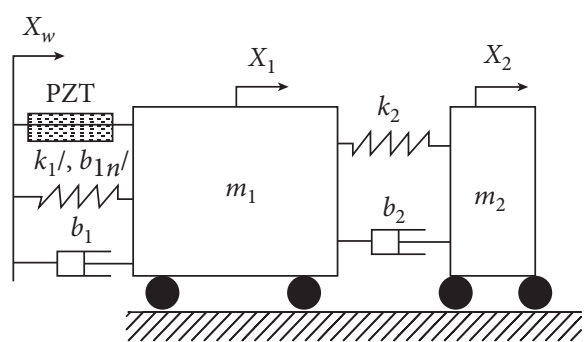

(c)

Figure 1: (a) The buoy system. (b) The portal frame structure. (c) Lumped parameter mode of the system.

where $\sigma_{1}$ and $\sigma_{2}$ are constants. As the external excitation is provided by the ocean waves, base excitation is considered as of harmonic kind which is given by $[15,16,22]$

$$
\begin{aligned}
& X_{w}=A_{\mathrm{mp}} \sin \left(2 \pi f_{1} t\right), \\
& \dot{X}_{w}=A_{\mathrm{mp}} 2 \pi f_{1} \cos \left(2 \pi f_{1} t\right),
\end{aligned}
$$

where $A_{\mathrm{mp}}$ is the amplitude of the ocean wave and $f_{1}$ is the main frequency of excitation. Hence, equations (2) and (3) are substituted into (1) and the new dimensionless parameters as considered as follows:

$$
\begin{aligned}
\omega_{1} & =\sqrt{\frac{k_{l 1}}{m_{1}}}, \\
\omega_{2} & =\sqrt{\frac{k_{2}}{m_{2}}}, \\
\alpha_{1} & =\frac{b_{1}}{\sqrt{k_{l 1} m_{1}}}, \\
\alpha_{2} & =\frac{m_{1} b_{2}}{m_{2}^{2} \omega_{1}}, \\
\Delta_{e} & =\frac{m_{1} g_{1}}{k_{l 1}}, \\
\beta_{1} & =\frac{k_{l 1}}{m_{1} \omega_{1}^{2}}, \\
\beta_{2} & =\frac{m_{1} k_{2}}{m_{2}^{2} \omega_{1}^{2}}, \\
\beta_{3} & =\frac{k_{l 1} \Delta_{e}^{2}}{k_{l 1}}, \\
\alpha_{4} & =\frac{A_{\mathrm{mp}}}{\Delta_{e}}, \\
\alpha_{5} & =\frac{2 \pi f_{1}}{\omega_{1}}, \\
\tau & =\omega_{1} t \\
\rho & =R C \omega_{1}, \\
X_{3} & =\frac{q}{q_{0}} .
\end{aligned}
$$
form:

Equations (1) and (3) can be represented in the following

$$
\begin{aligned}
\ddot{X}_{1}= & -\alpha_{1} \dot{\Delta}_{1}-\beta_{1} \Delta_{1}-\beta_{3} \Delta_{1}^{3}+\alpha_{2} \dot{\Delta}_{2}+\beta_{2} \Delta_{2} \\
& +\sigma_{1}\left(1+\sigma_{2}\left|X_{1}\right|\right) X_{3}, \\
\ddot{X}_{2}= & -\alpha_{2} \dot{\Delta}_{2}-\beta_{2} \Delta_{2}, \\
\dot{X}_{3}= & \frac{1}{\rho}\left[\sigma_{1}\left(1+\sigma_{2}\left|X_{1}\right|\right) X_{1}-X_{3}\right], \\
x_{w}= & \alpha_{4} \sin \left(\alpha_{5} \tau\right), \\
\dot{x}_{w}= & \alpha_{4} \alpha_{5} \cos \left(\alpha_{5} \tau\right) .
\end{aligned}
$$

Transforming equation (5) into state-space notation results in

$$
\begin{aligned}
\dot{x}_{1}= & x_{2}, \\
\dot{x}_{2}= & -\alpha_{1} \dot{\Delta}_{1}-\beta_{1} \Delta_{1}-\beta_{3} \Delta_{1}^{3}+\alpha_{2} \dot{\Delta}_{2}+\beta_{2} \Delta_{2} \\
& +\sigma_{1}\left(1+\sigma_{2}\left|x_{1}\right|\right) x_{5}, \\
\dot{x}_{3}= & x_{4}, \\
\dot{x}_{4}= & -\alpha_{2} \dot{\Delta}_{2}-\beta_{2} \Delta_{2}, \\
\dot{x}_{5}= & \frac{1}{\rho}\left[\sigma_{1}\left(1+\sigma_{2}\left|x_{1}\right|\right) x_{1}-x_{5}\right] .
\end{aligned}
$$

The harvested power is calculated according to the following equation:

$$
P_{\tau_{f}}=\sum_{\tau=0}^{\tau=\tau_{f}} \rho \dot{x}_{5}^{2},
$$

where $\tau_{f}$ is the time of the simulations.

\section{Particle Swarm Optimization}

The optimization of the energy harvesting is carried out by using particle swarm optimization (PSO). The PSO mimics the behavior of groups. Thus, the communication between each particle provides the survival of the crowdedness. 
Mathematically, each particle uses 3 factors, which are the local best (best for each group), the global best, and the inertia of each particle. This allows the introduction of a lag in each particle to avoid high changes in the velocity of the particles. As an iterative method, the displacement of the system can be calculated as [23-25]

$$
V_{n}^{i+1}=c_{0} V_{n}^{i}+c_{1} r_{1}\left(X_{n}^{i}-X_{\mathrm{gb}}^{i}\right)+c_{2} r_{2}\left(X_{n}^{i}-X_{\mathrm{lb}}^{i}\right)
$$

where $c_{0}$ is the inertia of the system, $V_{n}$ is the speed of the particle at the current state, $c_{1}$ is the self-knowledge, $r_{1}$ and $r_{2}$ are random values, $X_{\mathrm{lb}}$ is the direction for the local best, $X_{n}$ is the current position, $c_{2}$ is the group knowledge, $X_{\mathrm{gb}}$ is the direction for the group best, and $i$ is the iteration of the system.

\section{Optimal Control}

For the controller, the linear-quadratic regular (LQR) controller is chosen because it is multiple-input multipleoutput (MIMO) control that allocates poles optimized for the given restriction impose on the states and signal control [26]. The first step is to write the controlled system into statespace notation, in matrix form, as

$$
\dot{\mathbf{x}}=\mathbf{A x}+\mathbf{B u}+\mathbf{A}_{\mathrm{nl}},
$$

where $\mathbf{x}$ is the state vector, $\mathbf{A}$ is the state matrix for the linear, $\mathbf{B}$ is the input matrix, $\mathbf{u}$ is the control signal vector, and $\mathbf{A}_{\mathrm{nl}}$ is the nonlinear state matrix.

The control $\mathbf{u}$ law is given by

$$
\begin{aligned}
\mathbf{u} & =-\mathbf{R}^{-1} \mathbf{B}^{T} \mathbf{P e} \\
& =-\mathbf{k e},
\end{aligned}
$$

where $\mathbf{e}=\left[\begin{array}{lllll}\left(x_{1}-\tilde{x}_{1}\right) & \left(x_{2}-\tilde{x}_{2}\right) & \left(x_{3}-\tilde{x}_{3}\right) & \left(x_{4}-\tilde{x}_{4}\right) & \left(x_{5}-\tilde{x}_{5}\right)\end{array}\right]^{T}$ and $\tilde{x}_{s}$ represents the desired states for each $s$ state.

Being $\mathbf{P}$ a symmetric matrix, the algebraic Riccati equation is developed, which is denoted by

$$
\mathbf{P A}+\mathbf{A}^{T} \mathbf{P}-\mathbf{P Q R} \mathbf{R}^{-1} \mathbf{B}^{T} \mathbf{P}+\mathbf{Q}=0 .
$$

The control $\mathbf{u}$ is optimal and transfers the nonlinear system of equation (7) from any initial state to a final state $\mathbf{e}(\infty)=0$. Minimizing the cost functional, it yields

$$
J=\int_{0}^{\infty}\left(\mathbf{e}^{T} \mathbf{Q} \mathbf{e}+\mathbf{u}^{T} \mathbf{R} \mathbf{u}\right) \mathrm{d} \tau,
$$

where $\mathbf{Q}$ and $\mathbf{R}$ are positive definite matrices.

\section{Numerical Simulation}

The numerical simulations are carried out by integrating the system of equation (7) with the fourth-order Runge-Kutta method. The parameters accounted for in the numerical simulations are the ones described in Table 1.

In this way, we investigate the influence of some variables for the energy harvesting from the buoy system with the portal frame as the vibrating structure. In addition, the behavior of the neighborhood of these parameters is investigated and determined.
Parametric analyses for the dynamic behavior of the system and energy harvesting are carried out by varying specific nondimensional parameters of the system, such as damping of the TMD $\alpha_{2}$, the amplitude of the ocean waves $\alpha_{4}$, the linear stiffness of the TMD $\beta_{2}$, and the nonlinear stiffness of the portal frame structure $\beta_{3}$. The choice of these parameters is because they have a direct and strong influence on the dynamics of the system. It is expected that the TMD acts as an energy pump device, passively boosting extra vibration to the system. The parameter $\alpha_{2}$ directly changes the amplitude of vibration of the TMD, as well as $\beta_{2}$. However, $\beta_{2}$ tends to shift the natural frequency of the system and also may resonate with the portal frame movement, increasing the amplitude of vibration. Then, due to large deformations, nonlinear behavior can be observed as well as chaos at a low frequency depending on $\beta_{3}$. Offshore platforms are subjected to high amplitudes of vibration induced by the amplitude of the ocean waves (related to $\alpha_{4}$ ), which depends on its location in the ocean.

After the initial numerical simulations, the optimization process is carried out accounting for the optimization of the parameter vector $O=\left[\beta_{2}, \beta_{3}, \alpha_{2}, \alpha_{4}\right]$ restricted to the minimization of the function cost $J$ at $10000 / P_{1000}$ subjected to an interval of interest of the following parameters:

$$
\begin{gathered}
0.1<\beta_{2}<100 ; 0.1<\beta_{3}<10 ; \\
0.01<\alpha_{2}<0.2 ; 0.001<\alpha_{4}<1.5 ;
\end{gathered}
$$

where $P_{1000}$ is the power converter over a 1000 s. The chosen range is based on the mass of the TMD $m_{2}$, which affects $\alpha_{2}$ and $\beta_{2}$, the nonlinear stiffness $k_{\mathrm{nl}}$ for $\beta_{3}$, and the amplitude of the ocean waves $\left(A_{\mathrm{mp}}\right)$ based on data from NOAA [27]. Furthermore, numerical simulations with the optimized parameters are depicted and discussed.

5.1. Dynamics and Harvested Power Analysis. Using the parameters in Table 1 , the behavior of the buoy system is analyzed through the maximum Lyapunov exponent of the system, as illustrated in Figures 2(a) and 2(c). In addition, the average harvested power is calculated for the same region of parameters, as depicted in Figures 2(b) and 2(d).

Figure 2(a) presents the maximum Lyapunov exponent related to the amplitude of the wave exerted on the base of the system versus the damping coefficient of the TMD. It is observed that the higher is the damping, the system presents periodic behavior (gray area where $\lambda<-0.005$ ), except when the ocean wave amplitude is small (blue area where $0 \leq \lambda \leq-0.005)$. However, when damping is small $\left(\alpha_{2} \leq 0.5\right)$, irregular motions are noted for small and higher excitations. The values within the interval $0 \leq \lambda \leq-0.005$ represent an interval of transition between regular and irregular motions, which may represent from periodic, quasiperiodic, to chaos.

Figure 2(b) depicts the harvested power for the same interval of the parameters of Figure 2(a). It is observed that the harvested power is dominated by the excitation energy, which increases the harvested power from almost zero to $P_{\max }>500$. It is also highlighted that, although irregular motions can occur with high amplitudes of motion, for this 
TABle 1: Parameters of the system adapted from $[10,15,20]$.

\begin{tabular}{|c|c|c|c|c|c|c|c|}
\hline Parameter & Value & Parameter & Value & Parameter & Value & Parameter & Value \\
\hline$m_{1}(\mathrm{~kg})$ & 2 & $\alpha_{1}[]$ & 0.121 & $\beta_{1}[]$ & 1 & $k_{2}(\mathrm{~N} / \mathrm{m})$ & 43695.95 \\
\hline$k_{l 1}(\mathrm{~N} / \mathrm{m})$ & 8230.453 & $\alpha_{2}[]$ & 0.3916 & $\beta_{2}$ [] & 84.9 & $R(\Omega)$ & $1.55 \times 10^{4}$ \\
\hline$k_{n 1}\left(\mathrm{~N} / \mathrm{m}^{3}\right)$ & $k_{11} 10^{6}$ & $\alpha_{4}[]$ & 0.4195 & $\beta_{3}[]$ & 5.68 & $A_{m p}(\mathrm{~m})$ & 0.001 \\
\hline$b_{1}(\mathrm{~N} \cdot \mathrm{s} / \mathrm{m})$ & 1.55 & $\alpha_{5}[]$ & 0.0979 & $\sigma_{1}[]$ & 0.2 & $f_{1}(\mathrm{~Hz})$ & 1 \\
\hline$m_{2}(\mathrm{~kg})$ & 0.5 & $g\left(\mathrm{~m} / \mathrm{s}^{2}\right)$ & 9.81 & $\sigma_{2}[]$ & 0.6 & $b_{2}(\mathrm{~N} \cdot \mathrm{s} / \mathrm{m})$ & 3.14 \\
\hline$C(\mu \mathrm{F})$ & 1 & & & & & & \\
\hline
\end{tabular}

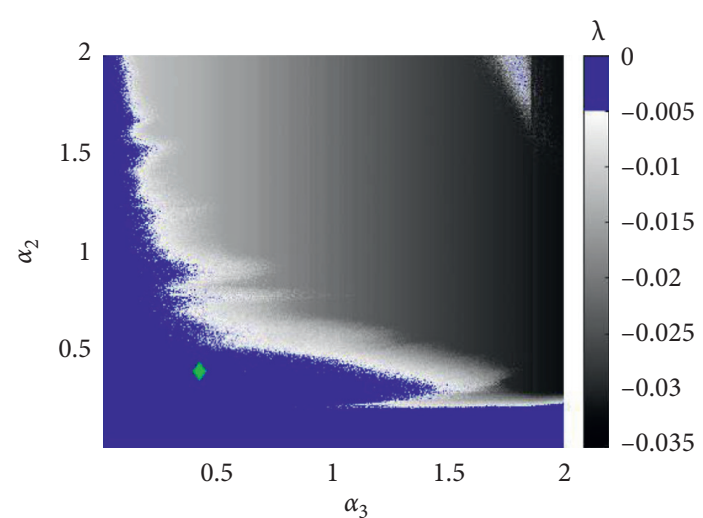

(a)

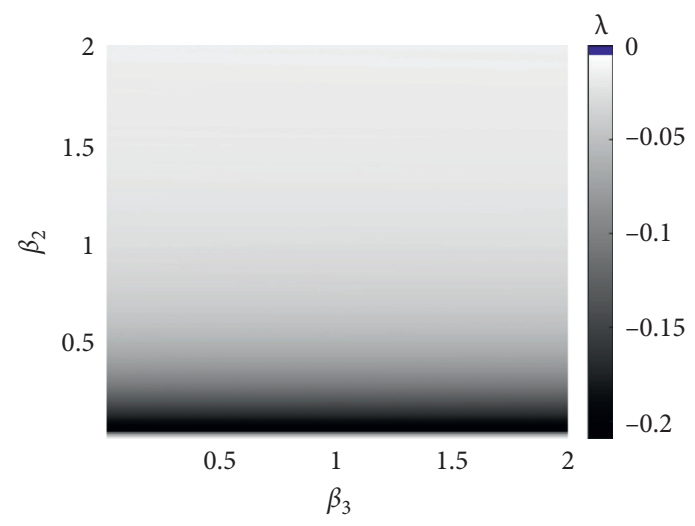

(c)

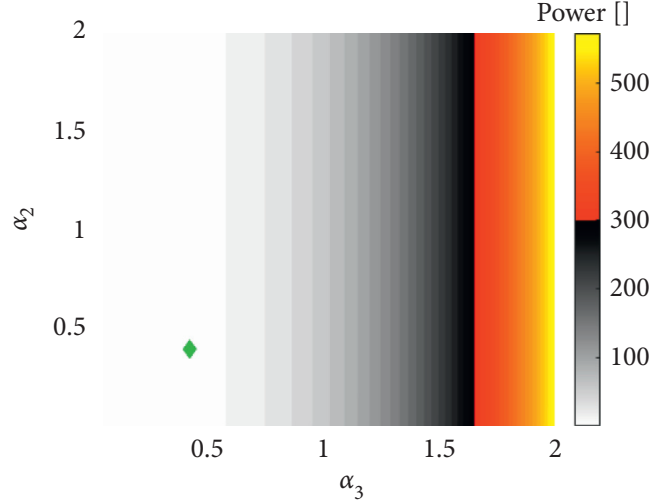

(b)

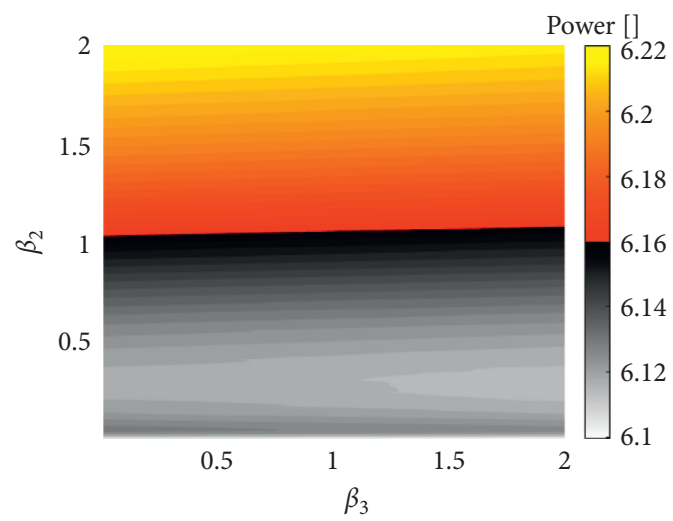

(d)

Figure 2: (a) Maximum Lyapunov exponent map for a parametrical variation of $\alpha_{2} \times \alpha_{4}$. (b) Average harvested power of the portal frame for $\alpha_{2} \times \alpha_{4}$. (c) Maximum Lyapunov exponent map for $\beta_{2} \times \beta_{3}$. (d) Average harvested power of the portal frame for $\beta_{2} \times \beta_{3}$.

current buoy system, it is of small amplitude (green diamond in Figures 2(a) and 2(b)). Consequently, the harvested power is very small, except for the system at low TMD damping and under high excitation.

Figure 2(c)) shows the maximum Lyapunov exponent for the parameters related to the linear stiffness of the TMD $\beta_{2}$ versus the nonlinear stiffness of the portal frame $\beta_{3}$. In this case, for the same range of parameters of Figure 2(a), there is no apparent irregular behavior. However, relating Figure 2(c) to its respective harvested power of Figure 2(d), it is noted that the harvested power increases with $\beta_{2}$ along with the increase of the maximum Lyapunov exponent. Although the Lyapunov exponent is less than zero, it seems the system is increasing the amplitude of displacement. The variation of the harvested power is very small within the interval of obtained power as $0 \leq P_{\text {avg }} \leq-0.005$.
5.2. Power Optimization. Establishing the most important parameters that the highest amount of energy is obtained, the optimization process using the PSO algorithm is carried out for the range of the parameters depicted in equation (14).

Figure 3(a) shows the maximum dimensionless harvested power obtained through the PSO algorithm for various swarm sizes. The number of swarm sizes indicates the number of particles used to find the set result. It is observed that the highest harvested power is found using only a swarm size of 24 , as highlighted by the blue bar. The optimized parameters found with the PSO algorithm for the highest harvested power are $\beta_{2}=0.1, \beta_{3}=0.1, \alpha_{2}=0.01$, and $\alpha_{4}=1.5$. Note that this result is the best among others using higher swarm sizes. This indicates that the highest harvested power is obtained for these found parameters and there is no other possible higher value in their 


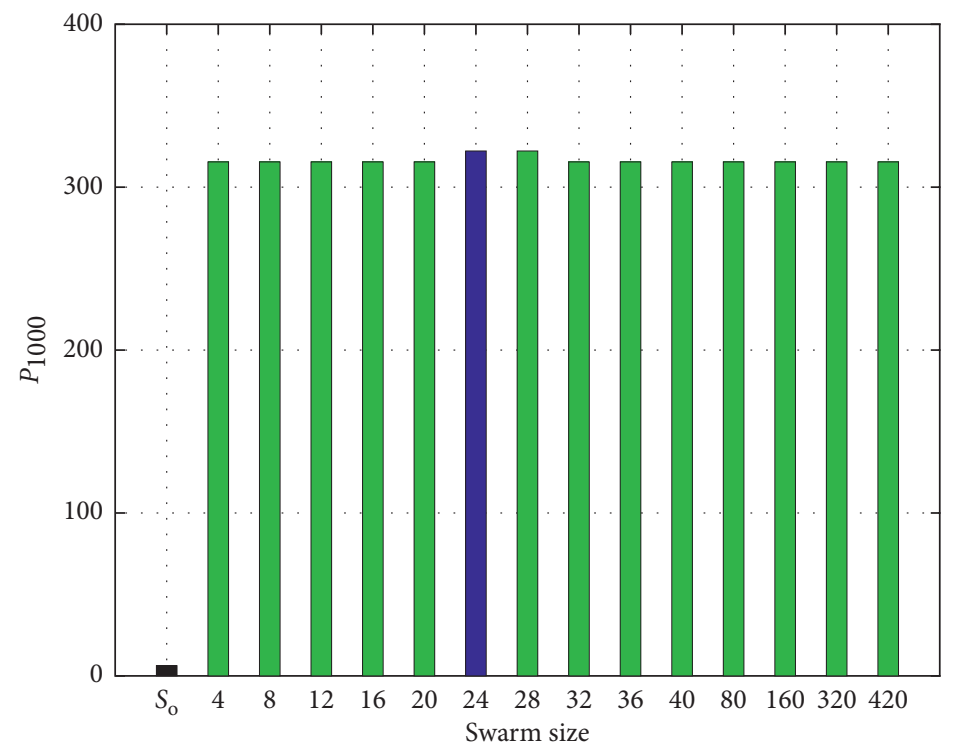

(a)

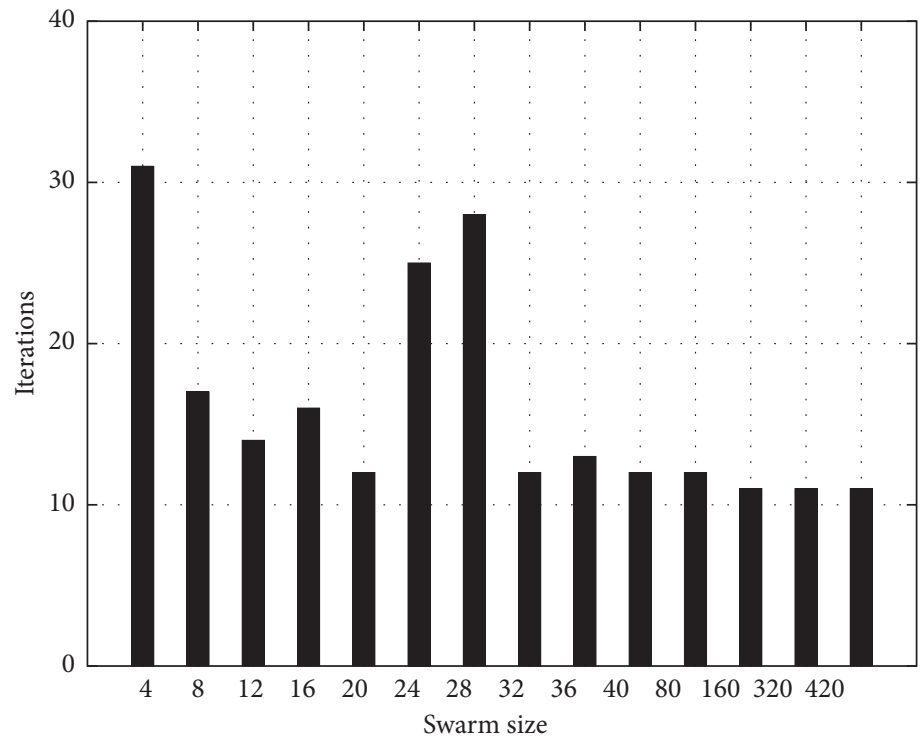

(b)

Figure 3: (a) Highest harvested power bar plots obtained for various swarm sizes. (b) Number of iterations for each swarm size. Both bar plots are obtained for a convergence time of $t=1000$.

neighborhood. However, the difference among all the obtained harvested power is almost insignificant.

Figure 3(b) shows the number of iterations for each swarm size. Note that the optimized result needed more iterations than the other results. However, it is expected due to the small number of particles of the PSO.

Figure 4 presents the number of iterations to minimize the function described by equation (14). During the PSO optimization process, all the solutions converged to the same value. It shows that, for this case, the size of the cluster does not affect the result. For a convergence with smaller cluster size, the convergence of the parameters has a satisfactory result.
On this hand, the dynamic behavior of the system and the harvested power are analyzed and calculated using equation (8) around the neighborhood of the optimized parameters, as depicted in Figure 5.

Figure 5(a) shows the map of the maximum Lyapunov exponent of the system for the variation of the dimensionless amplitude of the ocean waves $\alpha_{4}$ versus the damping coefficient of the TMD $\alpha_{2}$, accounting for the optimized $\beta_{2}$ and $\beta_{3}$, for the same intervals as in Figure 2. After the optimization, it is noted that the system becomes periodic in almost the full region of values (gray area where $\lambda<-0.005$ ). However, when damping is higher, the system may present quasiperiodic behavior and chaotic behavior. This is 


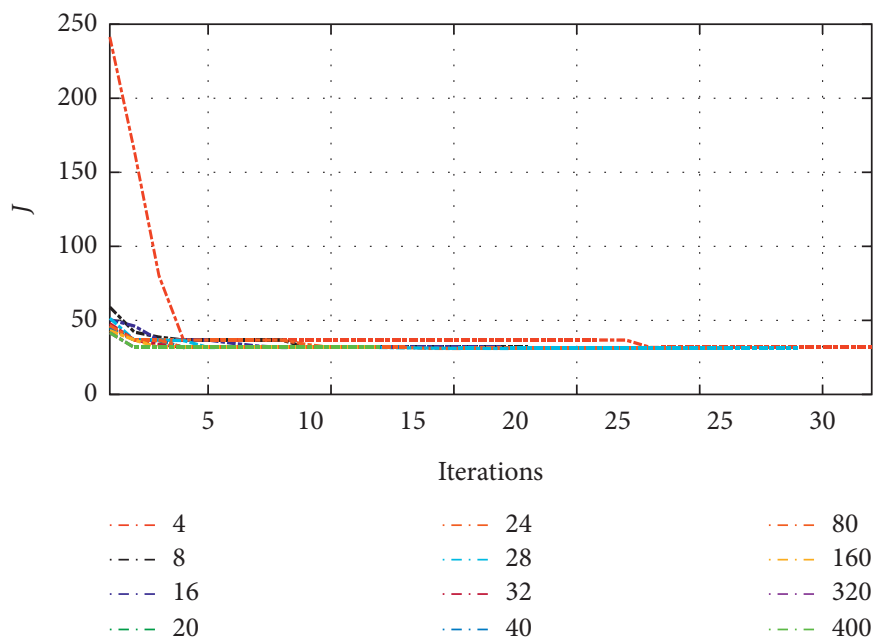

Figure 4: Plot of iterations for the convergence of equation (14), with the swarm number.

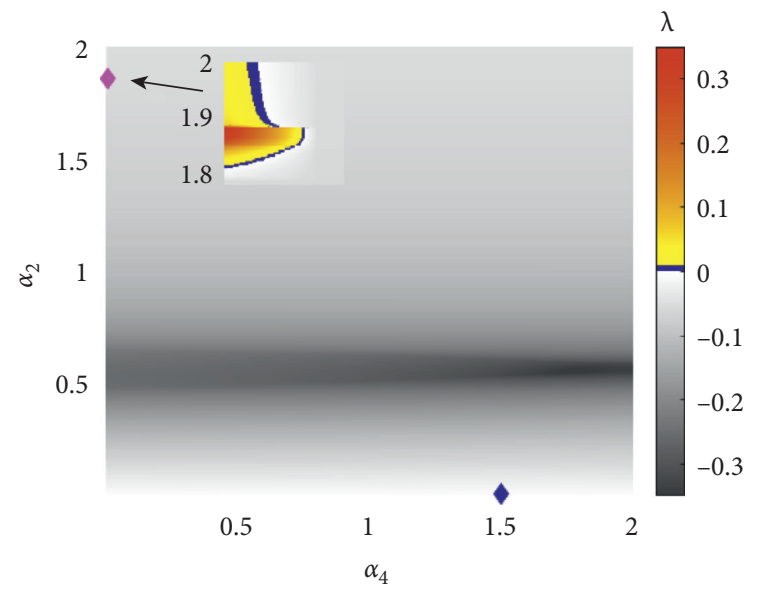

(a)

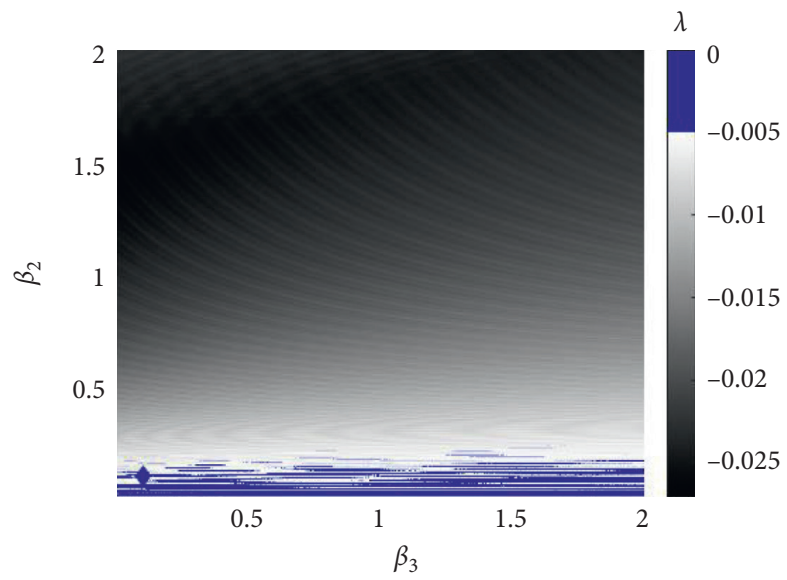

(c)

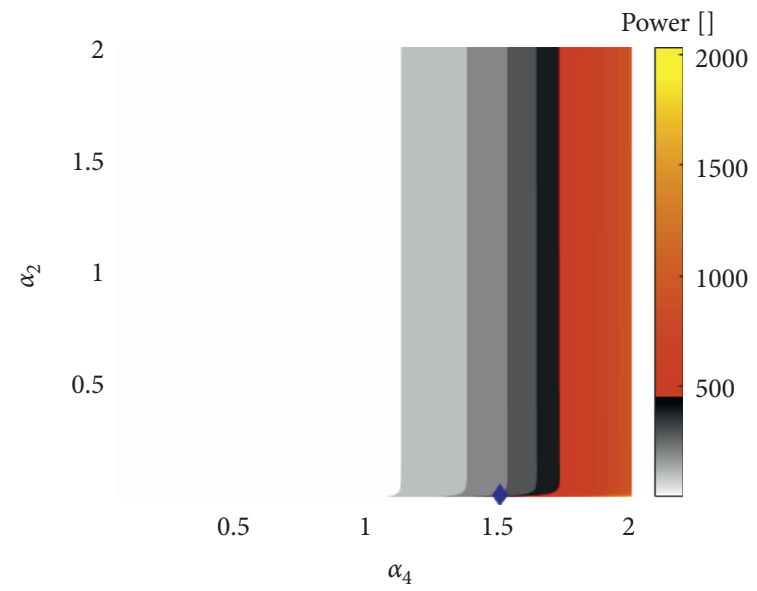

(b)

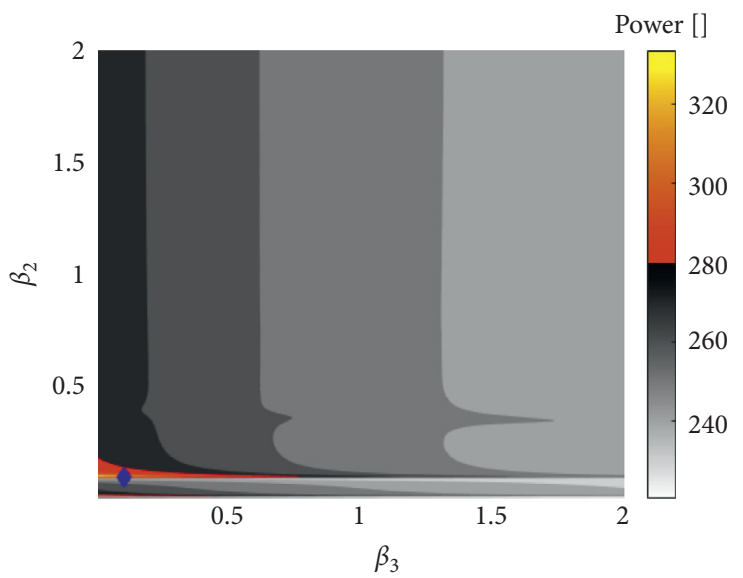

(d)

FIGURE 5: (a) Maximum Lyapunov exponent map for a parametrical variation of $\alpha_{2} \times \alpha_{4}$. (b) Average harvested power of the portal frame for $\alpha_{2} \times$ $\alpha_{4}$. (c) Maximum Lyapunov exponent map for $\beta_{2} \times \beta_{3}$. (d) Average harvested power of the portal frame for $\beta_{2} \times \beta_{3}$, for the optimized parameters.

highlighted by blue and red areas of the inset (purple diamond) of Figure 5(a), where the calculated maximum Lyapunov exponent is $\lambda=0$ (quasiperiodic or chaos) and $\lambda=0.3$ (chaos). The interval of the parameters of the inset is $1.8 \leq \alpha_{2} \leq 2.0$, and $0.001 \leq \alpha_{4} \leq 0.005$. With that, the unstable behaviors only occur for higher damping and low excitation. 
Figure 5(b) depicts the harvested power for the same interval of the parameters of Figure 5(a). It is observed that the maximum harvested power increases from around $P_{\max }=500$ to $P_{\max }=2000$, where the maximum power is obtained for the set of parameters represented in the blue diamond in the figure $\left(\alpha_{2}=0.01\right.$ and $\left.\alpha_{4}=1.5\right)$. Note that the same situation of the maximum harvested power, being at a periodic region and with high excitation amplitude, applies for the optimized parameters.

Figure 5(c) shows the maximum Lyapunov exponent for the parameters related to the linear stiffness of the TMD $\beta_{2}$ versus the nonlinear stiffness of the portal frame $\beta_{3}$, accounting for the optimized $\alpha_{2}$ and $\alpha_{4}$. In this case, for the same range of parameters of Figure 2(c), irregular motions (blue regions where $0 \leq \lambda \leq-0.005$ ) occur for the low linear stiffness of the TMD. It seems that the nonlinear stiffness of the portal frame becomes more dominant in the response of the system.

In addition, Figure 5(d) depicts the harvested power for the same region of Figure 5(c). A notable increase of the harvested power is observed in comparison with Figure 2(d), from $P_{\max }=6.18$ to $P_{\max }=333.8$ for $\beta_{2}=0.1$ and $\beta_{3}=0.1$. However, in this case, the maximum harvested power is obtained under an irregular motion that may be quasiperiodic or chaotic.

It is important to highlight that the PSO algorithm found the maximum harvested power for the abovementioned parameters with a very low computation task. Although it does not account for the analysis of the behavior of the system, it shows a neighborhood to be studied where it would take a long time to be optimized using other direct parametrical analyses.

5.3. Optimal Control Results. Considering equation (7) with the addition of a control signal ( $\mathbf{u})$, according to equation (10), the following matrices $\mathbf{A}$ and $\mathbf{B}$ are obtained:

$\mathbf{A}=\left[\begin{array}{ccccc}0 & 1 & 0 & 0 & 0 \\ -\beta_{1}-\beta_{2}-\beta_{3} x_{1}^{2} & \alpha_{1}-\alpha_{2} & \beta_{2} & \alpha_{2} & \sigma_{1}\left(1+\sigma_{2}\left|x_{1}\right|\right) \\ 0 & 0 & 0 & 1 & 0 \\ \beta_{2} & \alpha_{2} & -\beta_{2} & -\alpha_{2} & 0 \\ \frac{\sigma_{1}\left(1+\sigma_{2}\left|x_{1}\right|\right)}{R_{2} C \omega_{1}} & 0 & 0 & 0 & \frac{-1}{R_{2} C \omega_{1}}\end{array}\right]$,

$\mathbf{B}=\left[\begin{array}{l}0 \\ 0 \\ 0 \\ 0 \\ 1\end{array}\right]$.
Note from equation (15) that the matrix $\mathbf{A}$ is a function of $x_{1}$. Considering the particular case where $x_{1}=x_{1_{0}}=0$, the matrix $\mathbf{A}$ is obtained as

$$
\mathbf{A}=\left[\begin{array}{ccccc}
0 & 1 & 0 & 0 & 0 \\
-85.94 & -0.40 & 84.94 & 0.39 & 0.20 \\
0 & 0 & 0 & 1 & 0 \\
84.94 & 0.39 & -84.94 & -0.39 & 0 \\
0.20 & 0 & 0 & 0 & -1
\end{array}\right] .
$$

Next, the matrices $\mathbf{Q}$ and $\mathbf{R}$ are defined as follows:

$$
\begin{aligned}
& \mathbf{Q}=\left[\begin{array}{lllll}
1 & 0 & 0 & 0 & 0 \\
0 & 1 & 0 & 0 & 0 \\
0 & 0 & 1 & 0 & 0 \\
0 & 0 & 0 & 1 & 0 \\
0 & 0 & 0 & 0 & 1
\end{array}\right], \\
& \mathbf{R}=[1] .
\end{aligned}
$$

Replacing the matrices of equations (16) and (17) into equation (12), the vector gain $\mathbf{k}$ of the control law of equation (11) is obtained as follows:

$$
\mathbf{k}=\left[\begin{array}{lllll}
26.072 & 394.785 & 34.907 & 357.048 & 315.478
\end{array}\right] \text {. }
$$

The purpose of introducing a semiactive control by the LQR control is to enhance energy harvesting. In this way, to extract the maximum amount of energy, the desired variable $\tilde{x}_{5}$ is set as $\tilde{x}_{5}=x_{5}$, while $\tilde{x}_{1} \approx \tilde{x}_{2} \approx \tilde{x}_{3} \approx \tilde{x}_{4} \approx 0$.

To implement the control signal as an actuator, the introduction of a variable resistor in the electrical circuit of the piezoelectric system is considered, according to the following relationship:

$$
\begin{aligned}
\mathbf{u} & =R_{2} x_{5}, \\
\text { if: } x_{5} & \longrightarrow 0 ; \\
R_{2} & =0,
\end{aligned}
$$

where $R_{2}$ is obtained from

$$
R=R_{1}+R_{2}
$$

where $R$ is the general resistance, $R_{1}$ is the resistance of the circuit, and $R_{2}$ is a load resistance, which may be a battery or circuit to charge and exploit this energy.

To further increase the energy harvesting, the control is applied to the parameters obtained through the PSO.

Figure 6 shows the time histories of the TMD displacement $x_{1}$ (see Figure 6(a)), the portal frame displacement $x_{3}$ (see Figure $6(\mathrm{~b})$ ), and the voltage $x_{5}$ obtained through the piezoelectric material (see Figure 6(c)), accounting for the cases before the optimization, after the optimization, and with the control. For both cases with parameters obtained using the PSO and the system with control, an improvement of the voltage is obtained. It is also important to highlight that the control reduced the displacements of $x_{1}$ and $x_{3}$ and increased the variation of the 


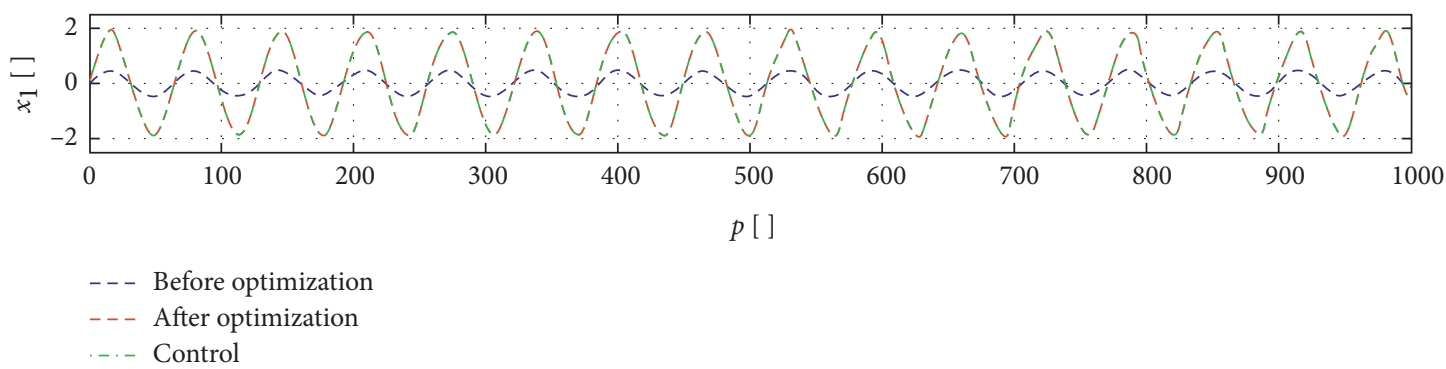

(a)

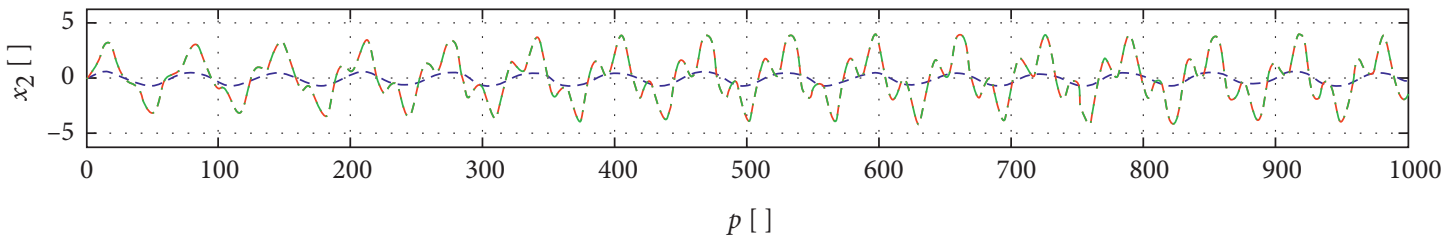

- - - Before optimization

- - - After optimization

-. - Control

(b)

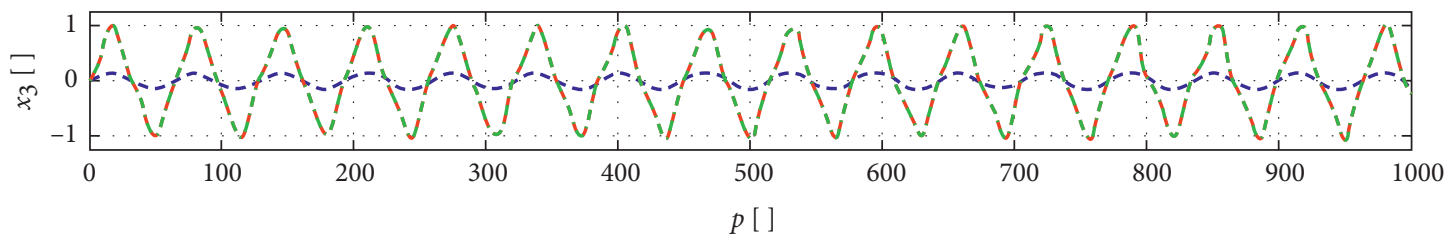

$\begin{array}{ll}\text { - - - } & \text { Before optimization } \\ \text { - - } & \text { After optimization } \\ \text {-.- } & \text { Control }\end{array}$

(c)

FIgURE 6: Time history of (a) the TMD displacement of $x_{1}$, (b) portal frame displacement $x_{3}$, and (c) voltage $x_{5}$. Figures showing results obtained before optimization, after optimization, and when the control is applied.

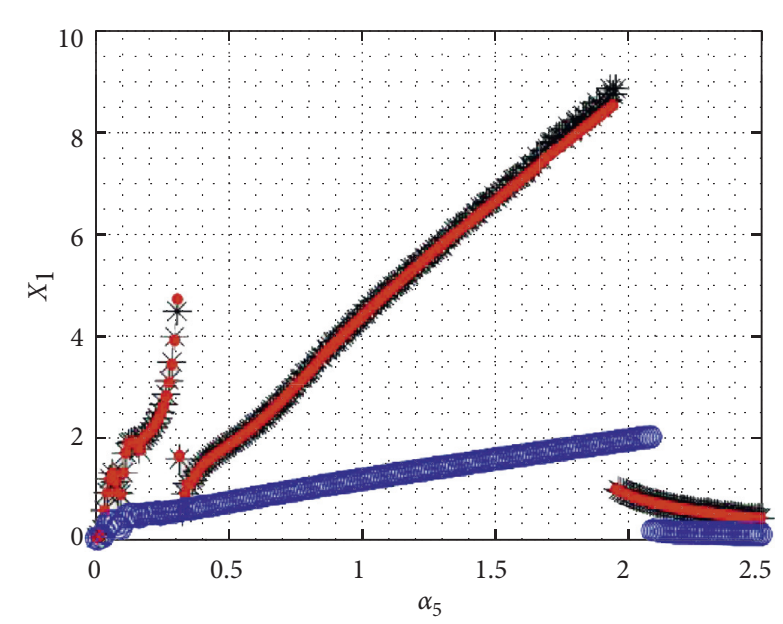

* Control

- Optimizated

Before opt.

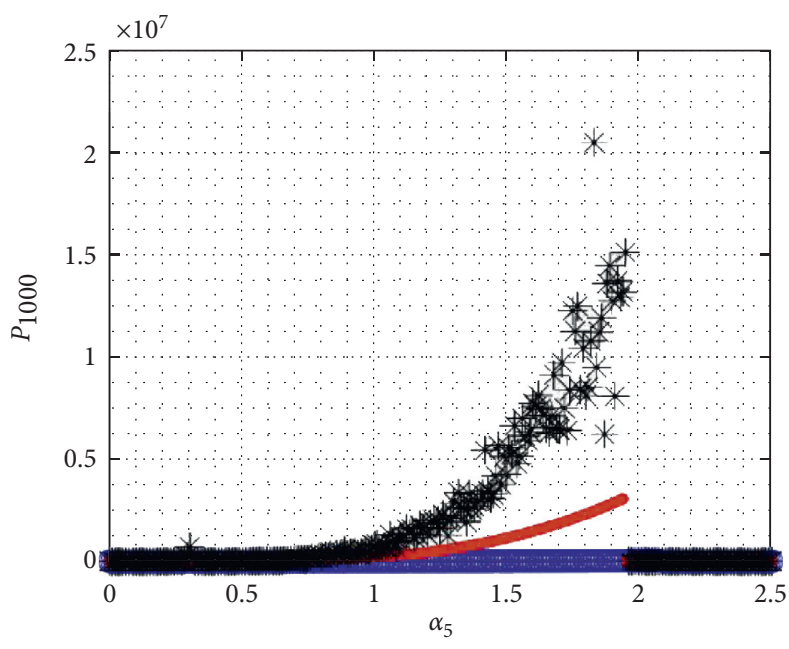

* Control

- Optimizated

Before opt.

(a)

(b)

Figure 7: (a) Frequency response of the portal frame excited around the resonance $0 \leq \alpha_{5} \leq 2.5\left(0 \leq f_{1} \leq 25.52\right.$ (Hz)), around low-frequency ocean waves. (b) Harvested power obtained through equation (8). Both results show the comparison before the optimization, after the optimization, and with control. 


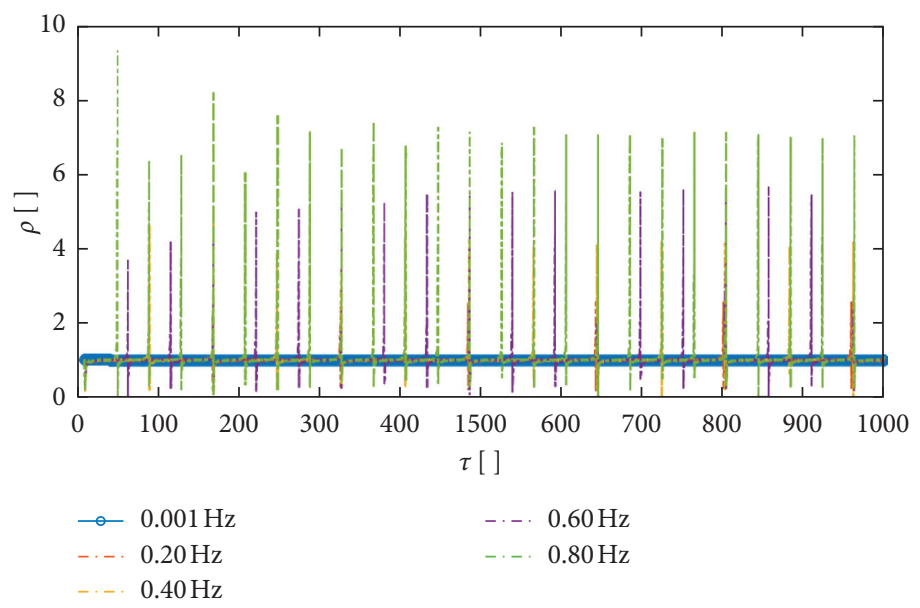

Figure 8: Variation $\rho$ in time.

voltage $x_{5}$, which are the desired states defined previously as $\tilde{x}_{5}=x_{5}$ and $\tilde{x}_{1} \approx \tilde{x}_{2} \approx \tilde{x}_{3} \approx \tilde{x}_{4} \approx 0$, demonstrating that the control proposal is very efficient.

In addition, when the system is set under the optimized parameters, it is observed the increase of the displacement of $x_{1}$ and $x_{3}$, which may be desired in many cases to increase the energy harvesting. However, it also may compromise the integrity of the structure due to large vibrations.

Figures 7 (a) and 7(b) show the frequency response and the energy harvesting of the system for the three cases varying the dimensionless excitation frequency $\left(\alpha_{5}\right)$ around the resonance, respectively. There is a notable difference between the harvested energy of the optimized system without and with control to the nonoptimized one, as illustrated in Figure 7(b). Note that energy harvesting increases with the increase of frequency. Due to the parametric excitation, the higher is the amplitude of excitation $\left(\alpha_{4}\right)$, the natural frequency shifts, and hardening nonlinear behavior is noted as the cubic nonlinearities dominate the response (see Figure 7(a) for the optimized parameters). When the control is applied, the harvested power is increased with the increase of the frequency. Although the control does not change the motion of the structure, it changes the resistance of the piezoelectric circuit, as the control is chosen to actuate it, varying the value of $\rho$, which is directly proportional to the harvested power.

It is also important to highlight that as the ocean waves are of low frequency due to their nature, the structure oscillates out of resonance. However, for a low frequency at $f=1 \mathrm{~Hz}\left(\alpha_{5}=0.0979\right)$, the optimized values and the control still make the system harvest a large amount of energy.

Figure 8 shows the variation of $\rho$ due to the resistance $R_{2}$ variation, according to the proposed control law (equation (18)). The proposed control strategy demonstrates to be feasible for applications in a real system, and that the increase of $\rho$ implies that the proposed control is consistent with equation (19).

\section{Conclusions}

This work proposed a model for an offshore energy harvesting application excited by ocean waves.
The PSO technique was used to analyze and optimize the parameters that maximize the energy production with a piezoelectric material for different conditions. The technique showed to be very efficient to optimize the energy harvesting obtained with the parameters $O_{\text {best }}=\left[\beta_{2}, \beta_{3}, \alpha_{2}, \alpha_{4}\right]=$ $[0.1,0.1,0.01,1.5]$. In addition, the results were obtained below the size of the cluster.

Therefore, we analyzed the behavior of the system in the neighborhood of these parameters with the maximum Lyapunov exponent, establishing the existence of chaotic behavior in it. However, chaos is found for the very low region with a low Lyapunov exponent, as seen in Figures 2 and 4 . In addition, chaotic behavior was found close to the optimized values (see Figure 4(c)).

A semiactive control applied by the LQR controller proved to be very effective in controlling the system and increasing the energy harvesting.

\section{Data Availability}

All data and information are available within the article. Additional data or information can be requested from the corresponding author.

\section{Conflicts of Interest}

The authors declare that there are no conflicts of interest regarding the publication of this article.

\section{Acknowledgments}

The authors acknowledge the CNPq, CAPES, FAPESP, and FA.

\section{References}

[1] S. F. Nabavi, A. Farshidianfar, and A. Afsharfard, "Novel piezoelectric-based ocean wave energy harvesting from offshore buoys," Applied Ocean Research, vol. 76, pp. 174-183, 2018.

[2] S. F. Nabavi, A. Farshidianfar, A. Afsharfard, and H. H. Khodaparast, "An ocean wave-based piezoelectric 
energy harvesting system using breaking wave force," International Journal of Mechanical Sciences, vol. 151, pp. 498-507, 2019.

[3] W. C. O’Reilly, T. H. C. Herbers, R. J. Seymour, and R. T. Guza, "A comparison of directional buoy and fixed platform measurements of Pacific swell," Journal of Atmospheric and Oceanic Technology, vol. 13, no. 1, pp. 231-238, 1996.

[4] A. F. d. O. Falcão, "Wave energy utilization: a review of the technologies," Renewable and Sustainable Energy Reviews, vol. 14, no. 3, pp. 899-918, 2010.

[5] K. Bønke and N. Ambli, "Prototype wave power stations in Norway," in Utilization of Ocean Waves-Wave to Energy Conversion, pp. 34-45, ASCE, Reston, VA, USA, 1986.

[6] V. C. Patil and P. I. Ro, "Energy and exergy analysis of ocean compressed air energy storage concepts," Journal of Engineering, vol. 2018, Article ID 5254102, 14 pages, 2018.

[7] R. Waters, M. Stålberg, O. Danielsson et al., "Experimental results from sea trials of an offshore wave energy system," Applied Physics Letters, vol. 90, no. 3, Article ID 034105, 2007.

[8] J. P. Kofoed, P. Frigaard, E. Friis-Madsen, and H. C. Sørensen, "Prototype testing of the wave energy converter wave dragon," Renewable Energy, vol. 31, no. 2, pp. 181-189, 2006.

[9] H. G. Xu, T. Ono, and M. Esashi, "Precise motion control of a nanopositioning PZT microstage using integrated capacitive displacement sensors," Journal of Micromechanics and Microengineering, vol. 16, no. 12, pp. 2747-2754, 2006.

[10] F. C. Janzen, A. M. Tusset, J. M. Balthazar, R. T. Rocha, J. J. De Lima, and A. Nabarrete, "Offshore energy harvesting of a marine floating pendulum platform model," Latin American Journal of Solids and Structures, vol. 16, pp. 1-13, 2019.

[11] A. Erturk and D. J. Inman, Piezoelectric Energy Harvesting, John Wiley \& Sons, Hoboken, NJ, USA, 2011.

[12] D. MaDeika, A. Heponis, and Y. Yang, "Multifrequency piezoelectric energy harvester based on polygon-shaped cantilever array," Shock and Vibration, vol. 2018, Article ID 5037187, 11 pages, 2018.

[13] Y. Zhao, Y. Qin, L. Guo, and B. Tang, "Modeling and experiment of a V-shaped piezoelectric energy harvester," Shock and Vibration, vol. 2018, Article ID 7082724, 15 pages, 2018.

[14] M. Yao, P. Liu, and H. Wang, "Nonlinear dynamics and power generation on a new bistable piezoelectric-electromagnetic energy harvester," Complexity, vol. 2020, Article ID 5681703, 29 pages, 2020.

[15] I. Iliuk, J. M. Balthazar, A. M. Tusset et al., "Application of passive control to energy harvester efficiency using a nonideal portal frame structural support system," Journal of Intelligent Material Systems and Structures, vol. 25, no. 4, pp. 417-429, 2014.

[16] I. Iliuk, R. M. L. R. da Fonseca Brasil, J. M. Balthazar, A. M. Tusset, V. Piccirillo, and J. R. C. Piqueira, "Potential application in energy harvesting of intermodal energy exchange in a frame: FEM analysis," International Journal of Structural Stability and Dynamics, vol. 14, no. 8, Article ID 1440027, 2014.

[17] K. H. Kim, S. B. Cho, H. D. Kim, and K. T. Shim, "Wave power generation by piezoelectric sensor attached to a coastal structure," Journal of Sensors, vol. 2018, Article ID 7986438, 7 pages, 2018

[18] Y. Shen and A. Homaifar, "Vibration control of flexible structures with PZT sensors and actuators," Journal of Vibration and Control, vol. 7, no. 3, pp. 417-451, 2001.
[19] A. Molter, O. A. A. da Silveira, J. S. O. Fonseca, and V. Bottega, "Simultaneous piezoelectric actuator and sensor placement optimization and control design of manipulators with flexible links using SDRE method," Mathematical Problems in Engineering, vol. 2010, Article ID 362437, 23 pages, 2010.

[20] R. T. Rocha, J. M. Balthazar, A. M. Tusset, V. Piccirillo, and J. L. P. Felix, "Nonlinear piezoelectric vibration energy harvesting from a portal frame with two-to-one internal resonance," Meccanica, vol. 52, no. 11-12, pp. 2583-2602, 2017.

[21] X. R. Chen, T. Q. Yang, W. Wang, and X. Yao, "Vibration energy harvesting with a clamped piezoelectric circular diaphragm," Ceramics International, vol. 38, pp. 271-274, 2012.

[22] W. H. Munk, Origin and Generation of Waves, Scripps Institution of Oceanography, La Jolla, CA, USA, 1951.

[23] R. Eberhart and J. Kennedy, "Particle swarm optimization," in Proceedings of the IEEE International Conference on Neural Networks, pp. 1942-1948, Perth, Australia, December 1995.

[24] K. E. Parsopoulos and M. N. Vrahatis, Particle Swarm Optimization and Intelligence: Advances and Applications, Information Science Publishing (IGI Global), Hershey, PA, USA, 2010.

[25] M. E. H. Pedersen, Good Parameters for Particle Swarm Optimization, pp. 1551-3203, Hvass Lab., Copenhagen, Denmark, 2010, Tech. Rep. HL1001.

[26] A. M. Tusset, V. Piccirillo, A. M. Bueno et al., "Chaos control and sensitivity analysis of a double pendulum arm excited by an RLC circuit based nonlinear shaker," Journal of Vibration and Control, vol. 22, pp. 3621-3637, 2016.

[27] National Oceanic and Armospheric Administration, https:// www.noaa.gov/, 2021. 